-

ISSN: 2178-7514

Vol. 13| No. 1| Ano 2021
ARTIGO ORIGINAL

\section{OS EFEITOS DA CARBOXITERAPIA NA GORDURA ABDOMINAL LOCALIZADA EM MULHERES JOVENS}

\section{The effects of carboxytherapy on abdominal fat located in young women}

Tereza Cristina dos Reis Ferreira ${ }^{1}$, Ana Karoline Sufredini ${ }^{2}$, Gabriela de Almeida Moraes ${ }^{2}$, Júlio César Veiga Pena ${ }^{3}$, Paula Tainah Soares Lima ${ }^{3}$, Paulo Vitor de Souza Sassim³, Antônio Gabriel Pantoja Silva Santos ${ }^{3}$, Gleidiane Lorrana Sales dos Santos ${ }^{3}$, Gabriel Vinícius Reis de Queiroz ${ }^{4}$, Raquel de Sousa Mota ${ }^{4}$

\title{
RESUMO
}

A gordura localizada abdominal é muito frequente em grande parte da população, provocando mudanças estéticas e se tornando cada vez mais uma grande preocupação com a aparência física, levando algumas pessoas a submeter-se a vários tipos de tratamento chegando até a optar por cirurgias plásticas. Esta gordura tem a função de proteger o organismo de traumas e do calor, depósito de calorias, permite a mobilidade da pele em relação às estruturas subjacentes e de modelar o corpo, sua distribuição vai depender de alguns fatores como sexo, hormônios e genética, assim o desenvolvimento destas células será influenciado pela restrição dietética e pelo exercício. A camada de gordura, ou panículo adiposo, é a camada mais profunda da pele sendo constituída de células adiposas com triglicerídeos em seu citoplasma. Para a redução da adiposidade abdominal a Fisioterapia Dermato-Funcional se dispõe de vários recursos como a carboxiterapia, eletrolipólise (eletrolipoforese), radiofreqüência, vacuoterapia e ultra-som, umas mais eficazes e outras ainda sem ter comprovação quanto aos seus efeitos. A carboxiterapia tem como efeito o aumento da oxigenação local, o aumento do tônus vascular e realiza uma vasodilatação ativa da microcirculação, favorecendo a lipólise. O efeito mecânico do gás carbônico também favorece a lise celular devido à velocidade e o fluxo com que este é penetrado, sendo substituído por fibras colágenas, elásticas e vasos sanguíneos. O presente estudo com a utilização de carboxiterapia na gordura localizada infra abdominal aplicando-se um fluxo de $80 \mathrm{ml} / \mathrm{min}$, volume de $480 \mathrm{ml}$, agulha no ângulo de $90^{\circ}$ (agulha de insulina medindo $0,30 \times 13 \mathrm{~mm}$ ) com variáveis analisadas circunferência e prega, os dados quando comparados o antes e o depois de 10 sessões, evidenciou-se que não foi obtida diferença estatisticamente significante na variável circunferência ( $\mathrm{p}=0.418)$ e na variável prega foi obtida diferença estatisticamente significante $(\mathrm{p}=0,0464)$.Acredita-se, desta forma, que serão necessários mais estudos para que o processo do tratamento voltado para a redução desta lipodistrofia sejam mais satisfatórios aumentando o número de sessões, o volume do gás, a aplicação da agulha em outro ângulo, utilizando um outro fluxo, adipometria na porção infra abdominal direita e esquerda e maior número de amostras, realizando também uma análise microscópica do tecido adiposo antes e a opós a utilização de carboxiterapia.

Palavras-chave: Gordura subcutânea Abdominal, Mulheres, Fisioterapia

\section{ABSTRACT}

Localized abdominal fat is very common in a large part of the population, causing aesthetic changes and becoming more and more a major concern with physical appearance, leading some people to undergo various types of treatment, even opting for plastic surgery. This fat has the function of protecting the body from trauma and heat, depositing calories, allowing the mobility of the skin in relation to the underlying structures and shaping the body, its distribution will depend on some factors such as sex, hormones and genetics, as well as the development of these cells will be influenced by dietary restriction and exercise. The fat layer, or adipose panicle, is the deepest layer of the skin, consisting of fat cells with triglycerides in their cytoplasm. To reduce abdominal adiposity, Dermato-Functional Physiotherapy has several resources, such as carboxitherapy, electrolipolysis (electrolipophoresis), radiofrequency, vacuotherapy and ultrasound, some of which are more effective and others without proof of their effects. Carboxitherapy has the effect of increasing local oxygenation, increasing vascular tone, and performs an active vasodilation of the microcirculation, favoring lipolysis. The mechanical effect of carbon dioxide also favors cell lysis due to the speed and flow with which it is penetrated, being replaced by collagen, elastic fibers and blood vessels. The present study with the use of carboxitherapy in the fat located below the abdomen applying a flow of $80 \mathrm{ml} / \mathrm{min}$, volume of $480 \mathrm{ml}$, needle at an angle of $90^{\circ}$ (insulin needle measuring 0.30 x $13 \mathrm{~mm}$ ) with variables analyzed: circumference and fold, the data when comparing the before and after 10 sessions, it was evidenced that no statistically significant difference was obtained in the variable circumference $(p=0.418)$ and in the variable fold was obtained statistically significant difference $(p=0.0464)$. It is believed, therefore, that more studies will be necessary for the treatment process aimed at reducing this lipodystrophy to be more satisfactory by increasing the number of sessions, the volume of the gas, the application of the needle at another angle, using another flow , adipometry in the right and left infra-abdominal portion and a larger number of samples, also performing a microscopic analysis of the adipose tissue before and after the use of carboxitherapy.

Keywords: Subcutaneous Fat, Abdominal, Women, Physical Therapy Specialty

1- Fisioterapeuta, Dra em Ciências da Reabilitação (UNINOVE)

2- Fisioterapeuta, Graduada pelo Centro Universitário do Pará (CESUPA)

3- Discente do Curso de Fisioterapia da Universidade do Estado do Pará (UEPA)

4- Fisioterapeuta, Graduado pela Universidade da Amazônia (UNAMA)

Autor de correspondência

Tereza Cristina dos Reis Ferreira

tereza.reis@uepa.br

DOI: $\underline{10.36692 / \mathrm{v} 13 \mathrm{n} 1-13}$ 


\section{INTRODUÇÃO}

A gordura localizada abdominal é muito frequente em grande parte da população, provocando mudanças estéticas e se tornando cada vez mais uma grande preocupação com a aparência física, levando algumas pessoas a submeter-se a vários tipos de tratamento chegando até a optar por cirurgias plásticas. ${ }^{1,2}$

Esta gordura tem a função de proteger o organismo de traumas e do calor, depósito de calorias, permite a mobilidade da pele em relação às estruturas subjacentes e de modelar o corpo, sua distribuição vai depender de alguns fatores como sexo, hormônios e genética, assim o desenvolvimento destas células será influenciado pela restrição dietética e pelo exercício. A camada de gordura, ou panículo adiposo, é a camada mais profunda da pele sendo constituída de células adiposas com triglicerídeos em seu citoplasma. ${ }^{3,4}$

Para a redução da adiposidade abdominal a Fisioterapia Dermatofuncional se dispõe de vários recursos como a carboxiterapia, eletrolipólise (eletrolipoforese), radiofreqüência, vacuoterapia e ultra-som, umas mais eficazes e outras ainda sem ter comprovação quanto aos seus efeitos. ${ }^{5}$

A fisioterapia Dermatofuncional tem uma grande área de atuação que antes era reconhecida apenas como área estética, com a função de melhorar ou restaurar a aparência, além disso, hoje ela restaura e melhora a função. Essa nova área já faz parte da Fisioterapia, se aprofundando cada vez mais no campo de pesquisa científica e tendo a responsabilidade de promoção de saúde ${ }^{4}$.

A carboxiterapia é uma técnica que vem sendo utilizada na Fisioterapia Dermatofuncional para o tratamento de estrias, fibro edema gelóide (celulite), lipodistrofia localizada, flacidez cutânea, cicatrizes, alopecias, entre outras ${ }^{6}$.

Este método utiliza a introdução percutânea de agulhas com CO2 (gás carbônico) no tecido celular subcutâneo, onde o mesmo é inodoro, incolor e atóxico, produzido pelo organismo diariamente e em grande quantidade, sendo eliminado pelos pulmões ${ }^{7,8,9}$.

A carboxiterapia tem como efeito o aumento da oxigenação local, o aumento do tônus vascular e realiza uma vasodilatação ativa da microcirculação, favorecendo a lipólise10. O efeito mecânico do gás carbônico também favorece a lise celular devido à velocidade e o fluxo com que este é penetrado, sendo substituído por fibras colágenas, elásticas e vasos sanguíneos ${ }^{11,12,13}$

Há algumas contra indicações da carboxiterapia como infarto agudo do miocárdio, angina instável, insuficiência cardíaca, hipertensão arterial, tromboflebite aguda, gangrena, infecções localizadas, epilepsia, insuficiência respiratória, insuficiência renal, gravidez, distúrbios psiquiátricos ${ }^{14,15}$. 


\section{MÉTODOS}

A pesquisa foi realizada após o aceite do orientador e do aceite da instituição, do termo de consentimento livre e esclarecido dos pacientes, e da submissão e aprovação do projeto pelo Comitê de Ética em Pesquisa do Centro Universitário do Pará, conforme as normas da resolução 196/96 do CNS.

O estudo em questão é do tipo experimental, quantitativo e prospectivo.

A captação das participantes foi feita por meio de anúncios fixados nos quadros de avisos das universidades.

A amostra intencional dessa pesquisa foi de 10 mulheres que possuíam lipodistrofia localizada na região infra-abdominal, porém durante o estudo houve a perca de 3 mulheres, totalizando no final 7 participantes. Em que foram incluídas na amostra de estudo mulheres com idade entre 20 e 30 anos.

Foram excluídas gestantes, mulheres com idade inferior a 20 anos e superior a 30 anos, as que apresentaram qualquer cardiopatia, tromboflebite aguda, gangrena, infecções localizadas, epilepsia, insuficiência respiratória, insuficiência renal, diabetes, distúrbios circulatórios, distúrbios psiquiátricos, as que fizeram cirurgia bariátrica e as que apresentaram ao longo do tratamento alteração de peso de $2 \mathrm{~kg}$ a mais ou $2 \mathrm{~kg}$ a menos.

As aplicações de carboxiterapia foram realizadas na clinicaACM AtaideClinic Center, localizada na Avenida Generalíssimo Deodoro, entre Conselheiro Furtado e Mundurucus, $\mathrm{n}^{\circ}$ 1887. Foram feitas 10 sessões de carboxiterapia realizada duas sessões por semana para cada paciente.

Inicialmente as pesquisadoras apresentaram e explicaram minuciosamente os objetivos (geral e específicos) do presente estudo para as pesquisadas, esclarecendo os métodos que seriam utilizados e pontuando os riscos e benefícios do trabalho. Depois de sanada todas as dúvidas e respondidos todos os questionamentos feitos pelas pesquisadas, as participante receberam o Termo de Consentimento Livre e Esclarecido (TCLE) e foram convidadas a assiná-lo caso concordassem com os termos dele.

Logo após foi realizado uma avaliação das participantes, pelas pesquisadoras, por meio de uma de uma ficha. Foi realizado a perimetria infra abdominal por meio de uma fita métrica estando a avaliada na posição ortostática, com os braços ao longo do corpo onde foi marcado com um lápis dermográfico $2 \mathrm{~cm}$ abaixo da cicatriz umbilical e posteriormente foi contornado toda região com a fita (FIGURA 1)

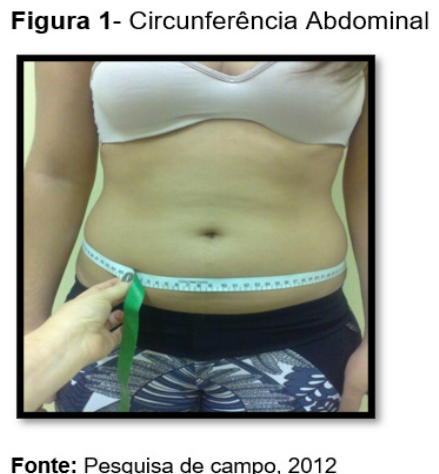

Fonte: Pesquisa de campo, 2012 
Seguindo o mesmo posicionamento foi realizado a quantificação da gordura na região infra abdominal com a utilização do adipômetro da marca cescorf, medindo- se $2 \mathrm{~cm}$ à direita da cicatriz umbilical, paralelamente ao eixo longitudinal do corpo (FIGURA 2).

Figura 2- Adipometria

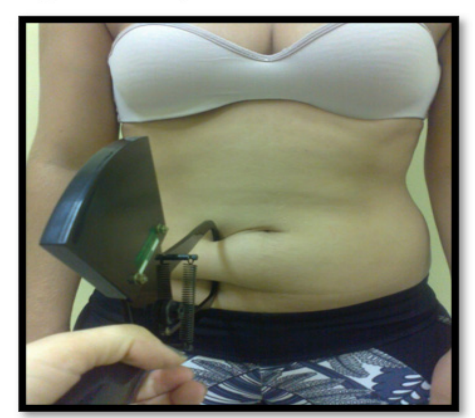

Fonte: Pesquisa de campo, 2012

Realizou- se a quantificação do peso da participante através da balança da marca welmey, estando a paciente de frente para balança, em que primeiramente se perguntava o peso médio da mesma. Posteriormente estando ela de costas era feito a mensuração da altura.

Antes do primeiro atendimento e após o último atendimento foram realizadas duas fotografias, para poder ser visualizado com maior clareza os resultados macroscópicos, não sendo anexadas no trabalho. Foi usado uma câmera da marca SONY que apresenta resolução 14,1 megapixels, onde as fotos foram tiradas há uma distância de $80 \mathrm{~cm}$, com uso de flash e a paciente estando de frente para a câmera na posição ortostática e com os braços ao longo do corpo, e de perfil direito e esquerdo estando a participante na posição ortostática e com os braços cruzados.
Antes de dar início ao primeiro atendimento do dia, era feito o esvaziamento de todo ar existente no equipo, com intuito de evitar a infusão de qualquer outro tipo de gás. A área de trabalho (região abdominal) a ser tratadas receberam assepsia e limpeza do local com álcool 70\% utilizando algodão. As pesquisadoras usaram jalecos, luvas de procedimentos e máscaras descartáveis, para evitarem qualquer contaminação. Antes e depois de qualquer conduta terapêutica realizaram higienização das mãos com sabão bactericida. Após a assepsia e limpeza do local a conduta terapêutica foi aplicada.

O aparelho utilizado foi CarboxiSkin, da marca Skiner (FIGURA 3), pertencente à ACM Ataide Clinic Center, onde as agulhas utilizadas foram abertas na presença da paciente, medindo 0,30 x $13 \mathrm{~mm}$ (agulha de insulina), sendo acopladas a uma cânula que era conectada ao aparelho de carboxiterapia e descartadas ao final de cada procedimento.

Figura 3- Aparelho de Carboxiterapia

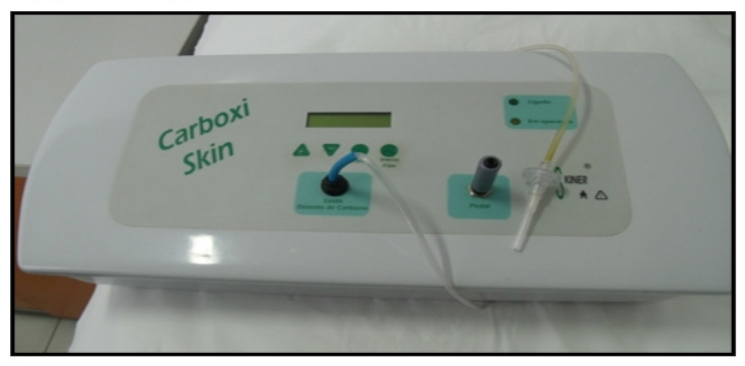

Fonte : Pesquisa de campo, 2012.

A agulha foi introduzida no plano hipodérmico, com uma inclinação de $90^{\circ}$. Realizado 4punturações de cada lado da região 
abdominal, totalizando 8 punturações, 4 na direção do umbigo e 4 na região infra-abdominal, e foi injetado o gás carbônico com um fluxo de $80 \mathrm{ml} / \mathrm{min}$, atingindo $480 \mathrm{ml} \mathrm{em}$ todo o tratamento. A paciente foi posicionada em uma posição confortável, onde a área a ser tratada ficava exposta e livre de qualquer pressão.

Ao final das 10 aplicações, foram realizadas reavaliações com as mesmas variáveis colhidas ao início das sessões.

Após a realização de todas as avaliações e reavaliações, as informações foram digitadas e tabuladas em banco de dados para análise estatística dos mesmos. De acordo com a natureza das variáveis, foram realizadas a análise estatística descritiva, sendo informados os valores percentuais dos resultados obtidos. O banco de dados, bem como as tabelas e os gráficos foram construídos no Microsoft EXCEL 2003. Para análise da significância estatística dos resultados obtidos foram utilizados testes estatísticos nãoparamétricos selecionados de acordo com a natureza das variáveis, sendo considerado o nível $\alpha=0,05(5 \%)$ sendo utilizado o softwareBioEstat $5.0^{\circledR}$, para análise descritiva dos dados.

\section{RESULTADOS}

Foram avaliadas 10 mulheres com lipodistrofia localizada infra-abdominal sendo que a casuística final constou de 7 mulheres, visto que a metodologia do estudo exigia a presença das pacientes duas vezes por semana, por isso foram excluídas 2 mulheres por faltaram mais de 3 sessões e 1 por motivo de doença. Com idade de 20 a 30 anos, sendo a média de idade de 22,2 anos, submetidas a 10 sessões de tratamento fisioterapeutico de carboxiterapia.As sessões foram feitas duas vezes por semana, totalizando 5 semanas de atendimentos e reavaliadas 3 dias após a última aplicação.

Em relação ao peso das participantes, a média inicial foi de $62,6 \mathrm{Kg}+11,3$ e média final de $62,3 \mathrm{Kg}$ $+11,2$; o que demonstra que as participantes não engordaram e nem emagreceram $2 \mathrm{~kg}$, permanencendo na média proposta pela metodologia do estudo.

A seguir apresenta-se os dados das variaveis analisadas antes e após as 10 sessões de carboxiterapia: Circunferência Abdominal e Prega Abdominal.

Em relação a circunferência abdominal a média inicial foi de $89,1 \mathrm{~cm}+8,6$ e a média final de $88,0 \mathrm{~cm}+8,1$. De acordo com o teste Wilcoxon não paramétrico para duas amostras relacionadas, a diferença média foi de $-1,1+-0,5$, obtendo $\mathrm{p}$ $=0.4185$, não sendo obtida diferença estatisticamente significante.

\begin{tabular}{|c|c|c|}
\hline Antes $(\mathrm{cm})$ & Depois $(\mathrm{cm})$ & Diferença $(\mathrm{cm})$ \\
\hline 87 & 87 & 0 \\
\hline 87 & 87 & 0 \\
\hline 104 & 103 & -1 \\
\hline 76 & 78 & 2 \\
\hline 94 & 88 & -6 \\
\hline 85 & 81 & -4 \\
\hline 91 & 92 & 1 \\
\hline
\end{tabular}


Quanto a variável prega abdominal -2,9+ -0,7, com nível de significância de 5\% ( $\mathrm{p} \leq$ obteve-se uma média inicial de $25,9+6,5$ e 0,05). A análise estatística em relação a variável média final de 23,0 + 5,8. De acordo com o prega abdominal demosntra que houve diferença teste Wilcoxon não paramétrico para duas estatisticamente significante por apresentar um amostras relacionadas, a diferença média foi de $\mathrm{p}=0,0464$ (TABELA 2).

\begin{tabular}{|c|c|c|}
\hline Antes $(\mathrm{mm})$ & $\operatorname{Depois}(\mathrm{mm})$ & Diferença $(\mathrm{mm})$ \\
\hline 24 & 25 & 1 \\
\hline 31 & 25 & -6 \\
\hline 38 & 34 & -4 \\
\hline 20 & 20 & 0 \\
\hline 20 & 16 & -4 \\
\hline 23 & 19 & -4 \\
\hline 25 & 22 & -3 \\
\hline
\end{tabular}

Fonte: Pesquisa de Campo,2012

Teste aplicado foi Wilcoxon: Teste não parametrico para duas amostras relacionada. p-valor $=(0.0464)$

\section{DISCUSSÃO}

Muitos métodos terapêuticos para o tratamento de redução da gordura localizada, são desenlvolvidos por este se tornar cada vez mais um grande problema estético, principlamente em países desenvolvidos e em desenvolvimento, com isso a carboxiterpia tem se apresentado como uma solução para este problema, mesmo que a base científica para este procedimento ainda não seja clara ${ }^{16}$.

No estudo realizado por Brandi et $\mathrm{al}^{17}$, avaliando o efeito da carboxiterapia no tratamento de adiposidades localizadas em coxas, joelhos e/ ou abdômen em 48 mulheres, foi observado uma redução na circunferência da partes submetidas à injeção subcutânea do gás carbônico e o efeito indireto da técnica na melhora da microcirculação e na ação lipolítica.

Em outro estudo de Brandi et $\mathrm{al}^{18}$, averiguaram o efeito da carboxiterapia no tratamento da irregulridade da pele pós lipoaspiração e na melhora da flacidez cutânea, onde obtiveram um resultado eficiente relatando que a técnica é segura, eficaz e sem grandes efeitos colaterias. Afirmando que a carboxiterapia tem como principal efeito a melhora da elasticidade e textura da pele.

No estudo de Goes ${ }^{19}$, ejetando gás carbônico em 20 pacientes de ambos os sexos, com faixa etária de 25 a 50 anos, com adiposidade abdominal, culotes e flancos, restringindo a alimentação, evitando bebidas alcóolicas e medicamentos, introdução de atividade física aeróbica de baixa intensidade e aumentando a ingestão de líquidos principalmente de água gelada. Obtiveram ao término da sexta sessão uma melhora na qualidade da pele, que se tornou mais firme e brilhante, melhora da imperfeição do contorno e redução de medidas nos flancos, abdômen e culotes. 
Hartmann, Bassenge, Hartmann ${ }^{20}$, revela em seu estudo que a carboxiterapia possue dois efeitos que se complementam. O primeiro é que o dióxido de carbono promove uma "lise" dos adipócitos mecanicamente, e em segundo lugar tem um efeito vasodilatador nos capilares da área onde foi feito o procedimento, aumentando o fluxo do local e por seguinte uma maior oxigenação. Tendo como um efeito final, menor quantidade de células de gordura e tecido subcutâneo mais firme, confirmando o resultado obtido neste estudo através da variável prega abdominal.

Devido o acúmulo de tecido adiposo apresentar eventos multifatoriais como fatores genéticos, alimentares, hábitos, atividades físicas, fatores de comorbidade e socioeconômicos, é quase impossível conseguir homogeneidade nos grupos a ser estudado ${ }^{16}$. Esta pode ser uma das razões pela qual este estudo não obteve resultado significativo na variável circunferência abdominal, onde é difícil o controle sobre as pacientes em relação ao nível de atividade física que executam, a qualidade dos alimentos que consomem, o hábido de vida que possuem, entre outros.

No estudo realizado por Costa et al ${ }^{21}$, com 15 mulheres, com idade entre 24 e 50 anos, apresentando acúmulo de gordura localizada na parede anterior do abdômen, foi verificado através da citometria computadorizada e através das medidas antropométricas de peso, circunferência umbilical e circunferência na crista ilíaca, se houve redução desta gordura comparando o antes e o depois da aplicação de carboxiterapia. Obtiveram resultados significativos no estudo histomorfológico dos adipócitos, havendo redução da área, diâmetro médio, perímetro, comprimento, largura e números dos adipócitos. Porém não se encontrou variação nas medidas de peso, circunferência umbilical e circunferência da crista ilíaca. Sugere-se que isto pode explicar o porquê que os resultados da variável circunferência infra-abdominal, deste presente estudo, não foi significativo.

O uso de CO2 percutâneo ainda é aplicada de foma empírica, não existindo estudos que padronizem esta técnica, como o volume necessário do gás carbônico e que demosnstrem os reais efeitos do CO2 no interior dos adipócitos ${ }^{22}$. Esta também pode ser umas das razões pela pouca significância deste estudo. Pois não se teve a certeza de que o volume, o fluxo e o número de sessões utilizados foram eficazes para o alcance da redução da lipodistrofia localizada abdominal.

\section{CONCLUSÃO}

\section{A Fisioterapia Dermatofuncional está} em crescimento, tendo uma grande importância na restauração da função e no aumento da autoestima das pessoas. Há poucos estudos que demonstrem os efeitos e resultados dos recursos utilizados na redução da lipodistrofia localizada infra-abdominal, entre eles a carboxiterapia. É muito escasso as pesquisas que mostrem os 
resultados da carboxiterapia na gordura localizada

sem estar associada a outros recursos.

O presente estudo com a utilização de carboxiterapia na gordura localizada infra abdominal aplicando-se um fluxo de $80 \mathrm{ml} /$ min, volume de $480 \mathrm{ml}$, agulha no ângulo de $90^{\circ}$ (agulha de insulina medindo 0,30 x $13 \mathrm{~mm}$ ) com variáveis analisadas circunferência e prega, os dados quando comparados o antes e o depois de 10 sessões, evidenciou-se que não foi obtida diferença estatisticamente significante na variável circunferência ( $p=0.418$ ) e na variável prega foi obtida diferença estatisticamente significante $(\mathrm{p}=$ 0,0464).

Acredita-se, desta forma, que serão necessários mais estudos para que o processo do tratamento voltado para a redução desta lipodistrofia sejam mais satisfatórios aumentando o número de sessões, o volume do gás, a aplicação da agulha em outro ângulo, utilizando um outro fluxo, adipometria na porção infra abdominal direita e esquerda e maior número de amostras, realizando também uma análise microscópica do tecido adiposo antes e a opós a utilização de carboxiterapia. Com isto os profissionais da área que façam o uso deste recurso em clínicas e consultórios tenham maior respaldo e que os clientes tenham resultados mais eficazes e concretos.

\section{REFERÊNCIAS}

1- MELO FILHO. Psicossomática Hoje.Porto Alegre: Artes Médicas, 1998.

2- PINTO, E.B.S; REYES, M.F.C; MARTINEZ, Y.P. Os Pioneiros e a Evolução. Rio de Janeiro: Revinter,1999. 3- AZULAY, R; AZULAY, D. Dermatologia. 4 ed. Rio de Janeiro: Guanabara Koogan. 2006, p.01-15.

4- GUIRRO, E. C. O; GUIRRO, R.RJ. Fisioterapia Dermato-Funcional: fundamentos, recursos, patologias. $3^{\mathrm{a}}$ ed. rev. e ampliada. Barueri, SP: Manole. 2004, p.0123

5- MEYER, P. F; OLIVEIRA, F.R.A; FUENTES, G.R; AGNE, J.E; SILVA, R.T.S; BARBOSA, A.M.Transdermoterapia por electroporaciónenlalipólisis abdominal .Recibidoel 7 de agosto de 2007; aceptadoel 13 de diciembre de 2007. Disponível em: http://www. elsevier.es/sites/default/files/elsevier/pdf/146/146v3 0n06a13131104pdf001.pdf.

Acessado em: 08/10/2011.

6- PAIVA, M.G; BOGGIO, R.F; PRADO, J.C; CARLUCCI, A.R.Protocolo para Tratamento da Alopecia Androgenética. Instituto Brasileiro de Pesquisa e Ensino-IBRAPE, São Paulo, Brasil. Disponível em: http://www.ibrape.com.br/site/artigos/alopecia_ androgenetica.pdf. Acessado em: 17/08/2011.

7- ITO, T; MOORE, J; KOSS, M.C. Topical Application of CO2 Increases Skin Blood Flow. JournalofInvestigativeDermatology. 1989; 93(2):259-62. Disponível em: http://www.nature.com/jid/journal/ v93/n2/pdf/5613271a.pdf. Acessado em: 20/09/2011. 8- GUYTON, A.C; HALL, J.E. Tratado de Fisiologia

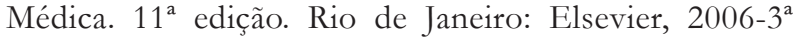
tiragem. p.495-512.

9- GANONG, W.F. Fisiologia Médica. 4ed. São Paulo. Atheneu, 2006.

10- KEDE, M.P.V; SABATOVICH, O. Dermatologia Estética. São Paulo: Atheneu. 2004, p. 03-08 e 349.

11 - KAFFER, E. N. Carboxiterapia: infusão terapêutica dermatológica de $\mathrm{CO} 2$ e tratamentos capilares, faciais, e corporais- novas práticas em Carboxiterapia capilar, facial e corporal. Produção: Assista, DVD 1, 2009 apud BORGES, F. S. Dermatofuncional: modalidades terapêuticas nas disfunções estéticas. São Paulo: Phorte. 2010.

12 - KAFFER, E. N. Carboxiterapia: infusão terapêutica dermatológica de $\mathrm{CO} 2$ e tratamentos capilares, faciais, e corporais- novas práticas em Carboxiterapia capilar, facial e corporal. Produção: Assista, DVD 4, 2009 apud BORGES, F. S. Dermatofuncional: modalidades terapêuticas nas disfunções estéticas. São Paulo: Phorte. 2010.

13 - LEGRAND, J.; BARTOLETTI, C.; PINTO, R. Manual práctico de medicina estética. Buenos Aires: Camarônes, 1999 apud BORGES, F. S. Dermatofuncional: modalidades terapêuticas nas disfunções estéticas. São Paulo: Phorte. 2010.

14- BROCKOW, T. et al. Clinical Evidence of Subcutaneous CO2 Insufflations: a systematic review. J. 
Altern. Complement. Med., v.6, n.5, p.391-403, oct.2000. Disponível em:

http://web.ebscohost.com/ehost/pdfviewer/pdf viewer?vid $=7 \&$ hid $=12 \&$ sid $=0316$ be 58-670d-4fe 2 9fde-a38a2e37129b\%40sessionmgr14. Acessado em: $11 / 07 / 2012$.

15- LEIBASCHOFF, G. Carboxytherapy. In: GOLDMAN, M.P; BACCI, P.A; LEIBASCHOFF, G; HEXSEL, D; ANGELINI, F.Cellulite Pathophysiology and Treatament. New York: Taylor \& Francis, 2006:197-208. Disponível em: http://www. drugs.am/upload/Cellulite\%20Pathophysiology $\% 20$ and $\% 20$ Treatment_1243340920375.pdf. Acessado em: 15/09/2011.

16 - BALIK, O.; YILMAX, M.; BAGRIYANIK, A. Does Carbon Dioxide Therapy Really Diminish Localized Adiposities? Experimental Study with Rats. Aesth Plast Surg (2011) 35:470-474. Disponível em: http://web.ebscohost.com/ehost/pdfviewer/pdfv iewer? vid $=5 \&$ hid $=127 \&$ sid $=3$ f135d04-6d $8 f-4660$ 97f0-96c6f41863d7\%40sessionmgr114. Acessado em: $12 / 07 / 2012$

17- BRANDI, C. et al .Carbon Dioxide Therapy in the Treatment of Localized Adiposities:Clinical Study and Histopathological Correlations.Aesthetic. Plast. Surg. , v. 25, n. 3. p. 170-174, may./jun. 2001. Disponível em: http://www.nutecint.com/Docs/APS\%20Article.pdf. Acessado em: 25/02/2012.

18- BRANDI, C. et al. Carbon Dioxide Therapy: Effects on Skin Irregularity and Its Use as a Complement to Liposuction. Aesth. Plast. Surg. 28:222-225, 2004. Disponível em: http://web.ebscohost.com/ehost/ pdfviewer $/$ pdfviewer? vid $=4 \&$ hid $=123 \&$ sid $=0$ eeb 5c46-9fd6-4313-aa61-29ca5fc3aacb\%40sessionmgr113. Acessado em: 25/02/2012

19- GOES, M.G.C. Carboxiterapia: Uma Experiência Surpreendente. Trabalho monográfico apresentado ao $1^{\circ}$ Capítulo Brasileiro de Medicina e Cirurgia Estética, Curso de Pós-graduação em Medicina e Cirurgia Estética. Salvador, 2005. Disponível em: http://carboxiterapia. tripod.com/ Acessado em: 15/08/2012.

20-HARTMANN, B.R.; BASSENGE, E.; HARTMANN, M. Effects of Serial Percutaneous Application of Carbon Dioxide in Intermittent Claudication: Results of a Controlled Trial. Angiology. 1997. Dísponivel em: http://web.ebscohost.com/ehost/pdfviewer/pd fviewer? vid $=8 \&$ hid $=127 \&$ sid $=3$ f135d04-6d $8 \mathrm{f}-4660$ 97f0-96c6f41863d7\%40sessionmgr114. Acessado em: 05/06/12.

21- COSTA et al. Avaliação Citométrica dos Adipócitos Localizados no Tecido Subcutâneo da Parede Anterior do Abdome após Infiltração Percutânea de CO2. Ver. Col. Bras. Cir. 2011; 38 (1); 015-023. Disponível em: http:/ / www.scielo.br/scielo.php? script=sci_arttex t\&pid=S0100-69912011000100004. Acessado em: $21 / 07 / 2012$

22- FERREIRA, J.C.T.; HADDAD, A.; TAVARES, S.A. Increase in collagen turnover induced by intradermal injection of carbon dioxide in rats. J Drugs Dermatol;
7(3): 201-6, 2008 Mar. Dísponivel em: http://www. ncbi.nlm.nih.gov/pubmed/18380201. Acessado em: 12/07/12

OBSERVAÇÃO: Os autores declaram não existir conflitos de interesse de qualquer natureza. 